\title{
Current Status of Methods Used In Degradation of Polymers: A Review
}

\author{
Aishwarya Kulkarni ${ }^{1}$ and Harshini Dasari ${ }^{1 *}$ \\ ${ }^{1}$ Department of Chemical Engineering, Manipal Institute of Technology, Manipal, Manipal Academy \\ of Higher Education, India.
}

\begin{abstract}
Degradation of different polymers now a day is the most crucial thing to carry out. It possesses threats to human health as well as to the environment. Different polymers like PVA, PVC, and PP with high density and low density are one of the most consumed by population and also their degradation is a bit difficult. For this many people have started working on effective methods of degradation of these polymers. This can be done by thermal degradation and pyrolysis which requires high temperature, bio degradation using starch, bacteria etc and photo degradation. Traditional gravimetric and respirometric techniques are the methods currently used in testing. They fit readily for degradable polymeric materials usually. Also they are well suited for biodegradable components with polymer blends. But the recent polymer generation is comparatively resistant to bio degradation of polymers hence they cannot be used here. The polymer matrices are readily present in the plasticizers boosting the strength of polymeric material hence in addition; there is the mechanism of degradation. The information on various methods discussed in this review is planned to illustrate a best fit of methods for those who are interested in testing the degradation of polymers under different environmental conditions and selection of appropriate technique for specific combination of mixture of polymer and catalysts which helps to degrade the polymeric material.
\end{abstract}

Keywords- Polymers, PVA, PP, PVC, thermal degradation, biodegradation, photo degradation, gravimetric.

\section{Introduction}

Chemical engineering involves new technologies employing highly novel materials whose unusual response at the molecular level endows them with unique properties. In recent years, polymers have gained an increased presence in the chemical industry with their highly defined properties and uses. Natural and synthetic polymers find a vital and pervasive use in day to day life [1] due to their versatile traits [2]. Polymers are the molecule whose structure contains multiple repeating units, which results into a compound of high relative molecular mass and associated properties[3].Polymers are widely used in petrochemical industries, oil

\footnotetext{
* Corresponding author: kulkarniaishwarya66@gmail.com
} 
\& gas companies, steel \& aluminium industries, plastic industries which involves bags, boxes etc. Also they find their adequacy in packaging of food items, agricultural produce, and electrical supplies and so on. Central Pollution Control Board stated that in 2017 plastic usage in India itself is 8,500,000 tonnes.

However, some of their most useful features, the chemical, physical and biological inertness, durability, low density, low cost has made their production efficient and easy, resulting in their accumulation in the environment if not recycled. The management of wastes generated by polymers is difficult whereas recycling can be feasible, environmentally and technically. For example waste generated by PE if sunken into yards, it causes adverse effect on soil which can lower the water drainage and agricultural produce will be reduced. Unfortunately, the accumulation of polymers has become a serious problem for all countries in the world. Therefore the need of hour is to use polymer that do not harm the environment or to find easy and energy efficient methods which helps to degrade them. Polymer degradation is the term used for process which depletes chemical and physical properties of polymer including their outward appearance. Various types of polymer degradation method including thermal, photo oxidative, mechanical, biological, by irradiation and chemical have been used traditionally. Ionic liquids have immersed as chemicals and solvents due to their unique properties and structural interactions for degradation. General mechanism for polymer degradation can be described briefly as follows.

\subsection{Mechanism of polymer degradation}

The major factors causing degradation are heat, mechanical energy, radiation and ozone leading to various types such as thermal, thermo oxidative, photo degradation, chemical, biological, hydrolytic and degradation by irradiation.

In a depolymerisation reaction, a reversal of polymerisation occurs which includes Initiation at chain ends, Depropogation, Termination.

\subsubsection{Depolymerization}

In depolymerisation process low activity free radical takes place at the end of polymer chain. The change in molecular chain takes lot of time as the monomers are lost by polymeric chain one by one as per the mechanism of chain reaction [4].

\subsubsection{Random chain scission}

Decrease in the molecular weight occurs rapidly as the backbone breaks down randomly which occurs at any site of backbone. Here two reactions namely disproportion termination and intermolecular chain transfer occurs resulting into formation of new free radicals having high reactivity.

\subsubsection{Side-group elimination}

Bonds connecting the backbone are much stronger than the bonds which connect the side groups to the backbone chain. When depolymerisation occurs side groups are removed from the backbone before any further breakage. 
For depolymerisation of PVC:

$$
\mathrm{CH}_{2}(\mathrm{Cl}) \mathrm{CHCH}_{2} \mathrm{CH}(\mathrm{Cl}) \rightarrow \mathrm{CH}=\mathrm{CH}-\mathrm{CH}=\mathrm{CH}+2 \mathrm{HCl}
$$

Depolymerisation of polymers is favoured when initiation by main chain depletion is possible or intermediates formed during the degradation reaction are stable or the activation energy required for the degradation reaction is very less. Therefore,

Activation energy of depropogation $=$ (activation energy of polymerization propagation $)+$ (heat of polymerization $\Delta \mathrm{H}_{\mathrm{p}}$ )

\section{Thermal degradation methods}

Polymer degradation and recycling is intended to prevent the environmental pollution caused by their production as they are often disposed in landfills or water. The long chain backbone of the polymer begins to separate and react at relatively elevated temperature.

Polymers have their own mechanism of degradation due to heat, light and chemicals. A few polymers are perceptive to oxidation and UV radiation. Whereas some are receptive to hydrolysis, acids and alkalis. Generally degradation reactions due to heat or thermal energy occur by depolymerization (main chain reactions) and substituent reactions. Depolymerization reaction occurs by breaking of main chain polymer which gives intermediate stage product which is similar to the parent polymer but the monomer units are distinguishable. This is called as unzipping. In substituent reactions the substituent's chemical nature is changed where as the chain structure remains the same [5]. Whereas by chemical energy which involves two techniques namely hydrolytic depolymerization which is considered to be an effective technique for chemical recycling of polymers and recovery of monomers and another as microwave irradiation which shortens the time required for degradation[6].

Tamer Karayildrum carried out a degradation of Polyvinylchloride, Polyethylene by stepwise pyrolysis using polymer combination with presence of $\mathrm{HCl}$ [7]. With the help of dynamic measurements, kinetic data for isothermal thermal decomposition of Polyethylene was done by Bockhorn et.al. Which proposed that pyrolysis with step by step process is possible if the sample of single polymer can be separated at particular temperature [8,9]. This degradation experiment was conducted in a cycled shape reactor as this reactor acts as a heat transfer agent. The extruder was kept at $190^{\circ} \mathrm{C}$ and polymer mixtures with any one of the polymer and catalyst was fed to reactor. Temperature was maintained at $330^{\circ} \mathrm{C}$. After the dechlorination, temperature was increased and then the mixture was held at $440^{\circ} \mathrm{C}$ for 60 min. Residue was collected from bottom. Then analysis was conducted like TGA and liquid analysis which showed that $\mathrm{CaCO}_{3}$ was most suitable for $\mathrm{HCl}$ fixation which produced liquid product. And $\mathrm{RM}$ was the most effective for $\mathrm{HCl}$ fixation and reduction.

A study for LDPE waste by thermal degradation was done by Ali Karaduman and Emir H. Simsek [10]. They suggested that this reaction is pseudo first order reaction and the reaction was completed in $30 \mathrm{~min}$ at $400^{\circ} \mathrm{C}$ and $425{ }^{\circ} \mathrm{C}$. Different models were generated for this experiment. 


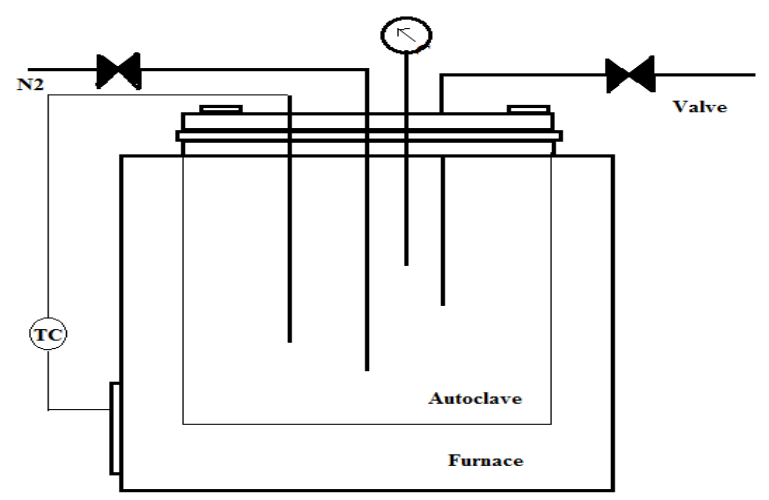

Fig. 1. Experimental set up.

Another method for thermal degradation was proposed by V.A.Kraikin et al [11]. They estimated that PTP, a heat resistant polyheteroarylene prone to carbonization and degradation of PTP begins at $480^{\circ} \mathrm{C}$. This process took place by decomposition of phthalide and evolution of $\mathrm{CO}$ and $\mathrm{CO}_{2}$. And $\mathrm{CH}_{4}$ and $\mathrm{H}_{2}$ and carbonization of polymer were at $500^{\circ} \mathrm{C}$. Degradation done by $\mathrm{CO}_{2}$ was about $12 \%$. Correlation between chemical structure and experimental data of molecular chains of polymer was studied by O.V.Okatova and N.N.Ul'yanova in 2007[12]. Polymer Poly (napthylimides) was thermally degraded with $96 \% \mathrm{H}_{2} \mathrm{SO}_{4}$ in PNI solutions which are observed to be stable and degradation occurred was $6.4-9.6 \%$. R.W.J.Westerhout proposed a method which is performed at elevated temperature typically from $300^{\circ} \mathrm{C}$ to $1000^{\circ} \mathrm{C}$ [13], for this high pressure equipment and solvents with high boiling point are required. To solve this issue, pyrolysis of polymers or supercritical water has been used in some cases. These type of reactions are usually performed in such apparatus which with stand the high pressure condition. Similarly in another experimental procedure developed by Akio Kamimuna, a 6-nylon chip having a molecular weight of 22,000 was reacted with a solvent at $300^{\circ} \mathrm{C}$ for one hour. Same conditions were provided under vacuum. The monomerized material was collected and checked for purity by GC-MS analysis. Depolymerization did not happen. $43 \%$ yield was gained after ionic liquid showed some preference [14].

However, polymers can be degraded by using ionic liquids as they offer specificity required to break the bonds at comparatively low temperatures. The most general and efficient methodology that may fulfill this requirement is phase transfer catalysis [PTC], studied by G.R. Gupta et al. Various reviews have been published on both engineering and chemistry view points of phase transfer catalyst in the last few decades. Cryptands, solvents, Onium salts, crown ethers, and co-solvents have been widely used as PTC [15]. Considering compatibility and relatively extensive solubility of ionic liquids with many inorganic and organic functional polymers it can also be used for dissolution of wood which comprise of cellulose, lignin, hemicelluloses and extractives. The complete acetylation of wood is done by ionic liquid solutions. Traditional ionic liquids were used to prepare by the metathesis reaction. Ionic liquids were used as catalytic solvents in dimeraization of 2-methylpropene to octane. Similarly for the depolymerization of PET such as aminolysis, hydrolysis, and alcoholysis have been put forward which in the absent of catalyst were very slow. The catalyst used were titanium phosphate, metal acetates, metal oxide, and solid super acids, 
sulphates, carbonates which were difficult to separate from the depolymerized products and disturbs the product analysis. Therefore it necessitated use of ionic liquids as studied by Martin J.Earle and Kenneth R.Seddon [16].

The products of all the reaction are insoluble in the ionic liquid and can be separated by decantation, leaving the ionic liquid and catalyst behind; whence the catalyst and solvent can be reused [17]. Use of ionic liquid for depolymerization of PVC stabilizes it by preventing the liberation of $\mathrm{HCl}$. While the PVC degrades rapidly therefore the solubilization and degradation depends upon structural characteristics of polymeric molecules [18]. Thermal degradation technique surely gives the desired output if correct parameters are chosen. But thermal degradation includes energy usage with very high demand. And for every industry it is not feasible to generate a degradation plant with high demand of energy and also it may involve evolution of gases.

\section{Biodegradation methods}

Four different mechanisms for study of biodegradation- charge formation, solubility, hydrolysis, enzymatic catalyzed degradation [19]. One of the major polymer, Polyethylene consumed is always thrown into environment. Until the olefins having high molecular weight were not found, polyethylene was assumed to be a non degradable by means of biodegradation. Vimala P.P. and Dr. Lea Mathew [20] in 2016 proposed a method which involves biodegradation of plastic. For this experiment they collected LDPE $(18 \mu)$ and HDPE $(19 \mu)$. Some sheets of polyethylene of specific thickness were exposed to UV treatment for some time. Bacillus subtilis which produces bio surfactant were also collected. Conical flasks were poured with bacterial species combined with polymer films, microbes and biosufactants. This was kept at room temperature till 30 days at $180 \mathrm{rpm}$ at about $32^{\circ} \mathrm{C}$. A weight loss of $9.36 \%$ was observed in $18 \mu \mathrm{PE}$ films. Formation of ketones, aldehydes, alcohols and carboxylic acids was observed. Weight loss after the UV treatment is listed as follows.

Table 1. Gravimetric analysis showing weight loss [20]

\begin{tabular}{|l|l|l|l|}
\hline Polymer Type & Before U V $(\mathrm{g})$ & After U V $(\mathrm{g})$ & Weight Loss $(\mathrm{g})$ \\
\hline $\mathrm{PE}(18 \mu)$ & 3.000 & 2.999 & 0.001 \\
\hline $\mathrm{PE}(41 \mu)$ & 3.000 & 3.000 & 0.000 \\
\hline
\end{tabular}

A similar approach was done in order to degrade polythene with the help of marine microorganisms mainly Bacillus pumilus, Bacillus subtilis and Kocuriapalustris. Polyethylene samples including bacterial strain which was incubated and another one which was non incubated. Based on properties like hydrobhobicity, FTIR analysis etc, most suitable bacteria was found for biodegradation. Properties showed the growth on surface of polymer with their metabolic activity giving rise to effective degradation and the most suitable bacteria found was Bacillus subtilis.

Another biodegradation methodology was put forward by K.S. Veethahavya, Rajath B.S. et al [21]. Biodegradation is the method in which the cost is very less as compared to any of the 
methods like thermal degradation or photocatalyatic degradation. The method proposed by them included degradation of low density polymer, polyethylene in liquid media. Starch content and polymer samples with different ratios were taken and a mixture containing aqueous media, ammonium nitrate, disodium hydrogen, and bacterial cells were subjected to a rotary shaker at room temperature. The results showed that with the proceeding number of days, starch content was also raised depending upon the combination used with polymer. Weight loss was observed from $1.53 \%$ up to $54.33 \%$ for pure low density polymer. For higher LDPE starch content, degradation achieved was higher and at lower levels, degradation was constant. $\mathrm{CO}_{2}$ was also evaluated from the bioreactor. The level was also stepped up with time.

From this experiment it is stated that at higher starch content, degradation was higher which was done by microorganisms as observed from HPLC analysis. Also, when there was degradation the peaks were vanished.

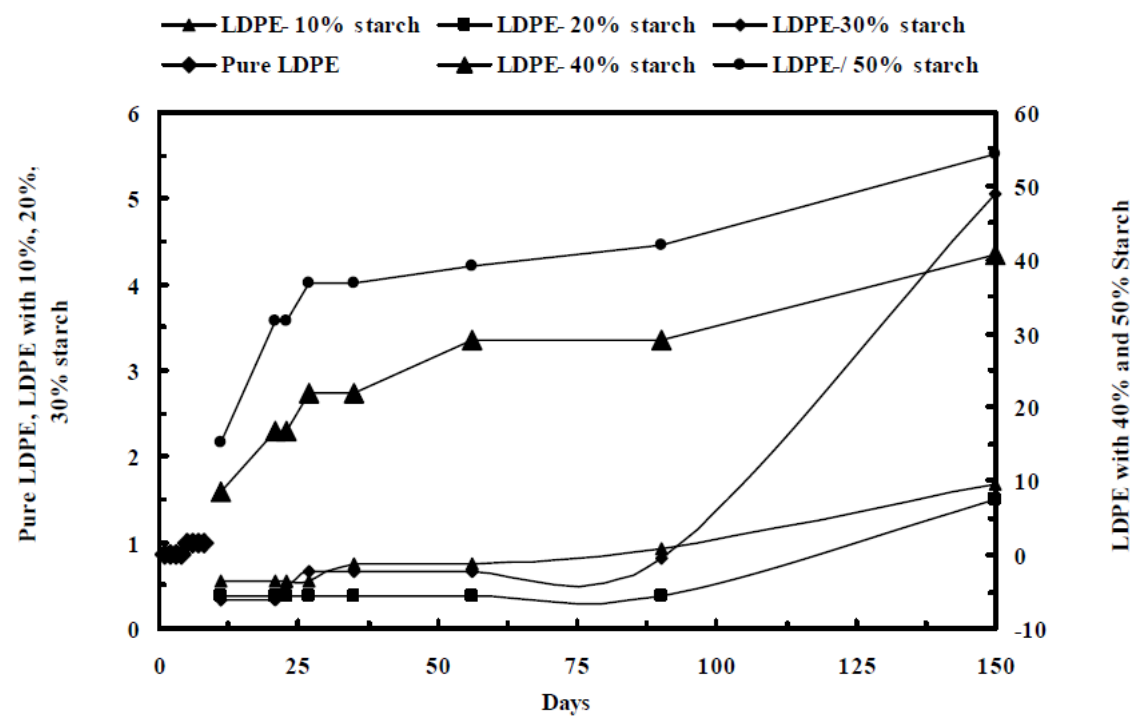

Fig. 3. Variation in percentage weight loss in aqueous media [21]

S. Schusser, M. Backer et al [22] suggested a technique for biodegradation using a field effect sensor base of which was semiconductor. This experiment involved a system which was monitored in a medium including Rhizomucormiehei which an enzyme lipase for degradation of poly (D,L-lactic acid). This polymer after spin coating gave a thickness of 500nm when solved with methyl ethyl ketone. Conductivity was maintained with $\mathrm{KCl}$ addition. Two different types of samples were prepared, one which was with lipase Rhizomucormiehei and another one with a buffer solution (pure) with $\mathrm{pH}$ 7. The results showed that polymer which was showing better degradation was suspected with buffer solution and the time required for this was about 12 days. For the mixture containing enzyme, sensor signal was reduced.

This same team proposed another system which was included with real time and also in-situ process degradation in 2015.It consisted of buffer solutions having different $\mathrm{pH}$ values, 
Rhizomucormiehei and a medium of cell culture. This time the lipase showed more rate of degradation than buffer solutions with $\mathrm{pH} 11$ and $\mathrm{pH}$ 7. But the buffer solution after addition of $\mathrm{NaOH}$ showed tremendous change in behaviour and degradation was favoured.

These experiments thus showed that the polymer degradation using sensor based system shows a great potntial indicating in situ and real time monitoring. Biodegradation methods are very econimic and they do not cause huge pollution as they involve treatment of polymeric mateials with micro-organisms. Hence this method is environmentally effective as there are no harmful effects. However it is bit difficult to get desired output as the microorganisums tend to affect slowly on the degradation process.

\section{Photo degaradation methods}

Photochemical degradation is another type of degradation which is used to degrade the polymer chains with UV rays and combinations used with different chemicals by means of thermal degradation. In the field of paints, art work poly vinyl acetate is generally used.For this experiment done by Shuya Wei, Valentina Pintus et al [23] different pigments were used. The samples to be treated were exposed to UV rays with $\mathrm{H} 2$ filter supported with a specific wavelengh range and ample radiation with temperature at $48.8^{\circ} \mathrm{C}$. For the analysis FTIRATR was used. It showed that the range obtained was abot 370 and $4000 / \mathrm{cm}$. Gas chromatography/ mass spectroscopy was used and different pyrolysis temperatures were attended. With increased amount of detactable acetic acid aged and uaged poly vinyl acetate paint samples were found and differentiated. The aged samples can be used for degradation as they can be used as effective indicators.

Another major polymer which is commonly found in textile waste water is poly vinyl alchohol(PVA) which is the major reason for incresed organic carbon content. For the degradation of this polymer, Diana Hamad, Ramdhane Dhib et al. [24] experimented photochemical degradation with contineous $\mathrm{UV} / \mathrm{H}_{2} \mathrm{O}_{2}$ process. Three aqueous solutions of PVA was prepared and mixed with a flask having distilled water. The solution was poured into a photo reactor. Hydrogen peroxide was added in intervals. Then sample was taken out after some specific time interval and molecular weight was tested along with total carbon content. Simultaneous exposure of feed to continuous flow of $\mathrm{UV}$ and $\mathrm{H}_{2} \mathrm{O}_{2}$ resulted in significant weight loss of polymer and total carbon content. The most significant factor was interaction between PVA and hydrogen peroxide affecting PVA degradation within the operating range of condition. 


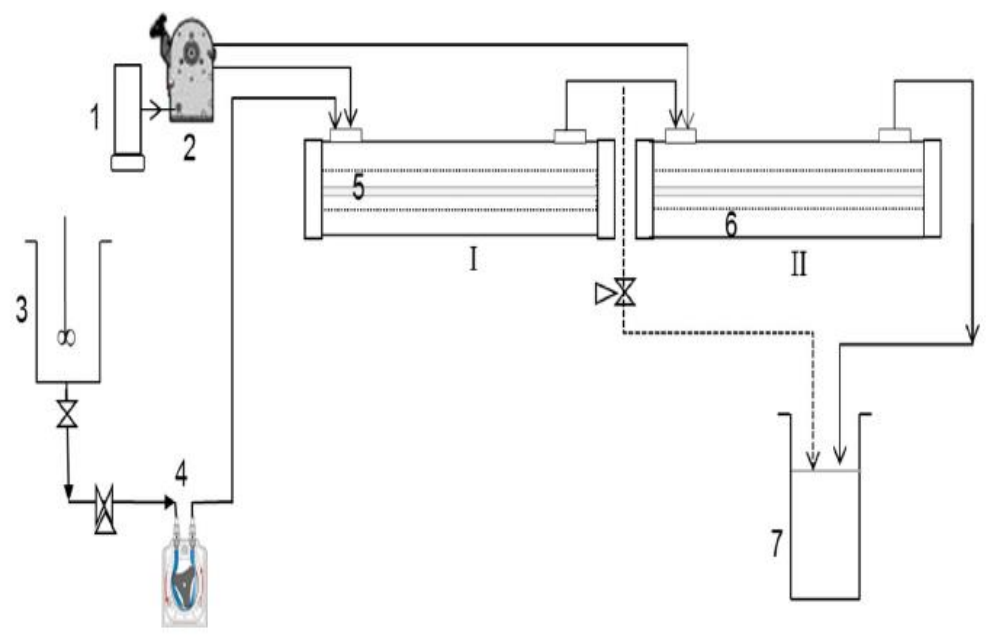

Fig. 5. Experimental setup for continuous $\mathrm{UV} / \mathrm{H}_{2} \mathrm{O}_{2}$ process [24].

Saba Sadaqat Ali, Ishtiaq A. Quazi et al [25] worked on degradation of LDPE (low density polymer) by using titania nanotubes with the help of photo catalytic degradation. For this degradation, PE was used which now a day's very hard to dispose and this is becoming a major issue. Photocatalyatic degradation in visible light is another effective method to use now days. Photocatalyst used here is titania nanotubes. The experimental setup included exposure of LDPE and composite films to UV light for 15 days and at room temperature. Again the experiment was carried out for 45 days. Each sample was weighted at regular time interval.

This study demonstrated photocatalytic degradation process of BG sensitized TNT composites PE films under visible light exposure. Dye sensitization of nanotubes has increased the degradation rate of LDPE films under visible light and those films can degrade under sunlight with good degradation rate hence it is more economical. Volatile organic product generation was found by FTIR, SEM and tensile strength procedures. This new LDPE-TNT material is environment friendly polymer material which could be degraded when exposed to the outdoor environment.

Telmo Ojeda, Ana Freitas et al. Studied the degradation of linear polyfins under natural weathering [26]. High density polyethylene, isotactic polypropylene and low density linear polythene having antioxidant additives at lower or zero levels were excluded and moulded as films by blowing. Mechanical properties and structural properties were studied by exposing these samples to natural weather conditions for one year. Weight loss of HDPE and LDPE was significant but the results were seen very slowly in a few months. Presence of pro oxidant additive and frequency of tertiary carbon atoms in polymer chain were the most influencing factors. Decline in degree of crystallinity after thermal destruction during exposure to natural weathering resulted by PP whereas increase in crystallinity was shown by HDPE/LLDPE oxo-biodegradable blend. 
Behaviour of three linear polymer samples poly methyl methacrylate (PMMA) having different molecular weights in a continuous ink jet printer is investigated by Joseph S.R. Wheeler, Stuart W. Reynolds et al [27]. The experimental setup is shown below in the figure. In this experiment the main source of polymer degradation is the continuous recycling of ink through pump resulting in mono chemical polymer degradation. Random scission achieves the molecular weight degradation and it found at lower shear rates. The delivery of high molecular weight polymers and materials sensitive to mechano-chemical degradation has the significance in total ink jet setup.

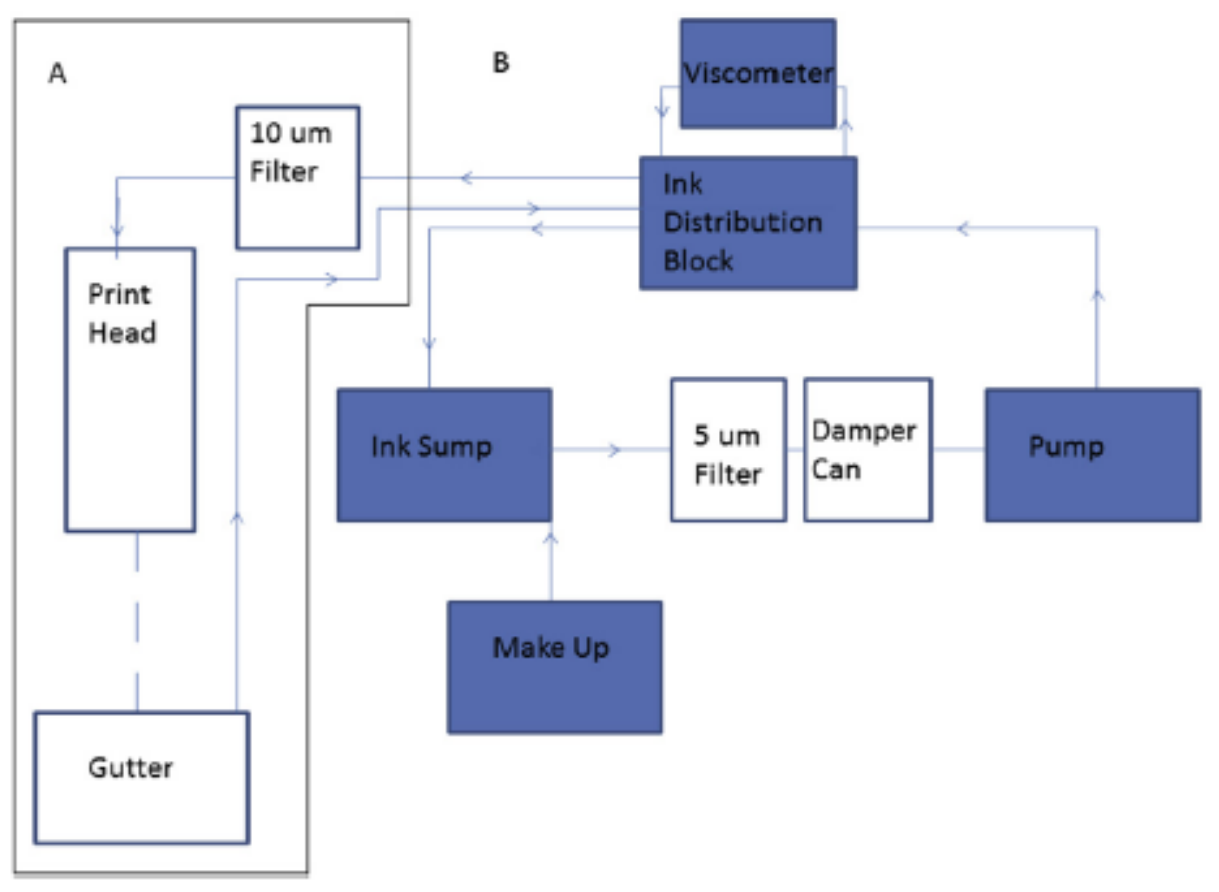

Fig. 6. Diagrammatic representation of ink jet system [27].

Photo degradation of polymers is one of the primary means by which the polymeric bonds can be broken. Generally in this method polymers tend to absorb energy radiations with high amount and it is not economical. But with certain limitations and proper selection of parameters, this method is useful.

\section{Conclusions}

In this study, different degradation models were proposed for degradation of polymers. Thermal degradation took place at very high temperatures and the time required for process was comparatively less than any other processes. The average temperature required for degradation with pyrolysis was around $400^{\circ} \mathrm{C}$. The degradation was done but the cost required for this and the heat economy was not suitable for all industries. Another method was to degrade polymers with microorganisms like Bacillus subtilis which was the most promising bacteria in order to degrade the polymers economically. But all the polymers can 
be degraded by this method as the environmental conditions are not suitable and also the time span is also large. Polymer degradation with photocatalytic and photo chemical degradation was studied which produced better degradation quality by using natural weathering and ink jet printers. The information will be helpful in control strategies in the extension of polymers' service life and formulation to recover it for better use.

\section{References}

1. N.G. McCrum, C.P. Buckley, C.B. Bucknall, Principles of polymer engineering. Oxford; New York: Oxford University Press. p.1.I SBN 0-19-856526-7(1997).

2. Paul C Painter, Michael M Coleman, Fundamentals of polymer science: An introductory text. Lancaster, Pa: Technomic Pub. Co. p.1.ISBN 1-56676-559-5. (1997).

3. http://goldbook.Iupac.org/Mo3667.html

4. http://nvl.nist.gov/pub/nistpubs/sp958-lide/344-346.pdf

5. A. Dharaskar Swapnil, Res.j.chem.sci. 2(8).80-85,August(2012)

6. Ahmad Adlie and DzulkeflyKuandAbdullah,"Ionic liquids: Preparations and limitations", makara,sains, 14, no.2, 101-106,(2010).

7. Tamer Karayildirim, JaleYanik, MithatYuksel, Mehmet Sagkam, and Michael Haussmann, J.Polym.Environ. 13, No.4, DOI: 10.1007/s10924-005-5531-2 (2005).

8. H. Backhorn, A.Hornung and U. Hornung, J.Anal.Appl.Pyrol.46,1-13

9. H. Backhorn, A.Hornung, U. Hornung,S. TeepeJ.Weichmann, Combust.sci.Technol 116/117, 129-151.

10. Ali Karaduman and Emir H. Simsek, , J.Polym.Environ.9, No.2,(2001).

11. V. A. Kraikin, Z.N.Musina, E.G.Galkin, S.I. Kuznetsov,A.E.Egorov, and S.N.Salzkin, J.Polym.Sci. A, 48,No.12,pp 1287-1303.(2016)

12. O.V.Okatova,N.N. Ul'yanova,E.G.Bilycheva,L.B.Elshia,A.L.Rusanov, and P.N. Lavrenko, J.Polym.Sci. A,49,No.6,pp 716-721.(2007)

13. R.W.J.Westerhout,J.Waanders,J.A.M.Kuipers, W.P.vanSwaaij.Ind.Eng.Chem.Res.37,2 993-2300(1998).

14. AkioKamimuna, ShigehiroYamamoto,Organic Letters.9,No.13,2553-2535. (2007).

15. G.R.Gupta,G.R.Choudhary,PreetiTomar,G.P.Waghulade,Kesharsingh J. Patil, "Synthesis of ionic liquids and derivatives using molten salt reaction media" Asian J.Chem.(2012)

16. Martyn J.Earle and Kenneth R.Seddon, Pure Appli. Chem.,72,No. 7,pp.13911398,(2000).

17. Q.F.Yue, C.X.Wang, L.N.Zhang, Y.Ni.Y.X.Jin, Polym Degrad Stab 96 399-403. (2011).

18. Preeti A.Tomar, S.M.Yadav and Gurav R.Gupta, "The thermal gravimetric studies for polymer samples of PVA and PVC obtained with treatment with ionic liquid [bmim]Br”,Polym. Bull, ISSN 0170-0839.

19. Gilding D.K., Biodegradable polymers. In:Williams, D.F.(Ed), Biocompatibility of clinic implant materials, 2 .CRC Prss, Boca Raton, FL.chapter 9.

20. P.P.Vimala and Lea Mathew, Proc. Technol.,24.232-239. (2016). 
21. K.S. Veethahavya, Rajath B.S., Sabike Noobia, B. Manoj Kumar, Procedia Environ Sci,35.709-713. (2016)

22. S. Schusser, M.Krischer,D.G.M.Molin,N.M.S.van den Akker, M.Backer, A. Poghossian, M.J.Schoning, Procedia Eng. 120, 948-951. (2015).

23. ShuyabWei, Valentina Pintus, Manfred Schreiner, Journal of Analytical and Applied Pyrolysis 97,158-163. (2012)

24. Dina Hamad, RamdhaneDhib,MehrabMehrwar, J Polym Environ,24:72-83,(2016).

25. SabaSadquat Ali, Ishiaq A. Quazi, Muhammad Arshad, Zahiruddin Khan, Thomas C. Voice,Ch. Tahir Mehmood, ENMM, 5, page no.44-53,(2016).

26. Telmao Ojeda, Ana Fretias, Katia Birk, EmileneDalmolin, Rodrigo Jacques, Fatima Bento, Flavio Camargo, Polym Degrad Stab,96,page no.703-707, (2011).

27. Joseph S.R.Wheeler, Stuart W. Reynolds, Steven Lancaster, Veronica Sanchez Romanguera, Stephen . Yeates, Polym Degrad Stab.105,page no.116-121, (2015). 